\title{
Effects of resistance training and detraining on muscle strength and blood lipid profiles in postmenopausal women
}

\author{
K J Elliott, C Sale, N T Cable
}

See end of article for authors' affiliations

......................

Correspondence to: Dr Cable, Research Institute for Sport and Exercise Sciences, Liverpool John Moores University, Henry Cotton Campus, Webster Street, Liverpool L3 2ET, UK; T.Cable@livjm.ac.uk

Accepted 25 February 2002

\begin{abstract}
Objectives: To study the effects of eight weeks of supervised, low intensity resistance training $180 \%$ of 10 repetition maximum (1ORM)) and eight weeks of detraining on muscle strength and blood lipid profiles in healthy, sedentary postmenopausal women.

Subjects: Fifteen postmenopausal women, aged 49-62 years, took part in the study. Subjects were assigned to either a control $(n=7)$ or training $(n=8)$ group. The training regimen consisted of three sets of eight repetitions of leg press, bench press, knee extension, knee flexion, and lat pull-down, three days a week at $80 \%$ of 10 RM. Dynamic leg strength, 10RM, and blood lipid profiles (total cholesterol (TC), low and high density lipoprotein cholesterol (LDL-C, HDL-C), triglycerides, and very low density lipoprotein cholesterol (VLDL-C)) were measured at baseline, after eight weeks of training, and after a further eight weeks of detraining.

Results: Eight weeks of resistance training produced significant increases in knee extension $\left(F_{1,13}=\right.$ $12.60 ; p<0.01)$, bench press $\left(F_{1,13}=13.79 ; p<0.01\right)$, leg press $\left(F_{1,13}=15.65 ; p<0.01\right)$, and lat pulldown $\left(F_{1,13}=16.60 ; p<0.005\right)$ 1ORM strength tests. Although 10RM strength decreased after eight weeks of detraining, the results remained significantly elevated from baseline measures. Eight weeks of training did not result in any significant alterations in blood lipid profiles, body composition, or dynamic isokinetic leg strength. There were no significant differences in any of the variables investigated over the 16 week period in the control group.

Conclusions: These data suggest that a short, low intensity resistance training programme produces substantial improvements in muscle strength. Training of this intensity and duration was not sufficient to produce significant alterations in blood lipid concentrations.
\end{abstract}

A ge related muscle weakness is a well accepted and reported phenomenon ${ }^{12}$ caused by muscle atrophy and specific weakening of the remaining muscle. Muscle atrophy is a coalition of functional disuse and an alteration in muscle composition. Adults who do not perform regular resistance exercise lose approximately $0.46 \mathrm{~kg}$ of muscle per annum from the fifth decade. ${ }^{3}$ Furthermore, sedentary subjects have reported a 50\% reduction in type II muscle fibres, the fibres responsible for high levels of strength, by age 80 years. ${ }^{4}$

Depletion of sex hormone has been hypothesised as the cause of specific muscle weakness, as women experience a dramatic reduction in muscle strength after the menopause. ${ }^{1}$ Strength can be maintained or increased at this time by the administration of hormone replacement therapy (HRT)..$^{5-6}$ Alternatively, resistance exercise can be used as a potential method of offsetting any decline in muscle strength after the menopause, as significant improvements in muscle strength have been shown in elderly women after resistance training. ${ }^{7-9}$ This is of particular relevance to patients with oestrogen dependent cancers, active trombophlebitis, or thromboembolic disorders, liver disease, or Dubin-Johnson and Rotor syndromes, for whom HRT use is contraindicated. ${ }^{10}$

In addition to specific muscle weakness, negative changes in blood lipid profiles are associated with advancing age and alterations in reproductive function, ${ }^{11}$ which combined have serious implications for women's health. After the menopause, oestrogen levels decline causing adverse effects on serum lipids and lipoproteins. ${ }^{12}{ }^{13}$ Oestrogen replacement therapy has been consistently shown to increase high density lipoprotein cholesterol (HDL-C), while concomitantly reducing serum total and low density lipoprotein (LDL) cholesterol, in postmenopausal women. ${ }^{14}$ Previous research has also examined the links between aerobic exercise and positive alterations to the blood lipid profile..$^{15-17}$ Prabhakaran et al ${ }^{18}$ examined the effect of resistance training ( 14 weeks training at $85 \%$ of 1 RM) on blood lipid profiles in premenopausal women. They found a significant decrease in total cholesterol (TC), LDL-C and the TC/HDL-C ratio, but no significant differences in HDL-C or triglyceride concentrations. Both epidemiological and clinical investigations have shown that lower TC and LDL-C levels, combined with raised HDL-C levels, are associated with a reduced risk of coronary heart disease. ${ }^{19-21}$ Bemben and Bemben ${ }^{22}$ observed raised HDL-C concentrations in hormone depleted postmenopausal women after a 16 week Dynaband training programme. With the exception of these studies, the impact of resistance training on blood lipid profiles in women has been largely ignored, particularly in the postmenopausal population who have an increased risk of cardiovascular disease.

Therefore the purpose of this study was to examine the effects of a low intensity, progressive resistance training programme (eight weeks supervised resistance training, three days a week at $80 \%$ of 10RM) on muscle strength and blood lipid profiles in postmenopausal women depleted of sex hormone. The effect of eight weeks of detraining was also investigated.

Abbreviations: HRT, hormone replacement therapy; 1ORM, ten repetition maximum; HDL-C, high density lipoprotein cholesterol; LDL-C, low density lipoprotein cholesterol; TC, total cholesterol; VLD-C, very low density lipoprotein cholesterol; BMI, body mass index 

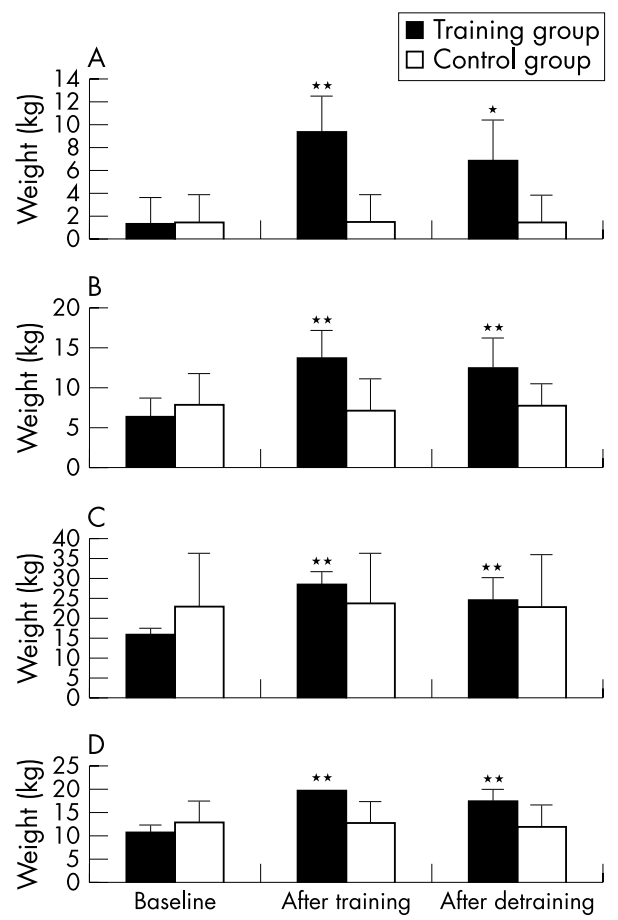

Figure 1 Ten repetition maximum (1ORM) strength for knee extension (A), bench press (B), leg press (C), and lat pull down (D) measured at baseline, after training, and after detraining for the training and control groups. Values are mean (SD) *Significant difference from baseline at the $p<0.01$ level; ** significant difference from baseline at the $p<0.005$ level.

\section{MATERIALS AND METHODS \\ Subjects}

Twenty five sedentary, postmenopausal women volunteered to participate in this study. Eight dropped out for personal reasons and two were excluded because of high blood pressure. Subjects were considered to be sedentary if they did not report any physical activity in excess of "everyday household tasks". ${ }^{23}$ Postmenopausal status (more than one year of amenorrhoea) was confirmed by blood sampling $(<150$ $\mathrm{pmol} / \mathrm{l}$ oestradiol; $>20$ units/l follicle stimulating hormone and luteinising hormone). Subjects were randomly assigned to either a training or a control group: eight in the training group (mean (SD) age 58 (4) years) and seven in the control group (mean (SD) age 53 (3) years). They were matched for years since menopause (5 (3) years in training group and 4 (3) years in control group).

Subjects were not receiving HRT or any other medication known to alter muscle strength. In addition, all subjects were free from myopathic, neuropathic, skeletal, thrombotic, or cardiovascular disorders. Approval for the experimental protocol was obtained from the institution's research ethics committee. All subjects provided written consent having read and understood the details of the experiment.

\section{Experimental design}

Before the experiment, all subjects were familiarised with the laboratory environment and experimental procedures. Both groups were then tested on three occasions (baseline, after the intervention, and after eight weeks of detraining). Women in the training group exercised three days a week for eight consecutive weeks, and those in the control group maintained sedentary physical activity levels. Subjects had no alcohol or caffeine for 24 hours before the tests, which were performed at least three hours after a meal. In addition, the training group was not tested within 48 hours of the previous training session. All testing and training took place at the same time of day to control for circadian variation in performance. Subjects showed $100 \%$ compliance with exercise training.

\section{Measurement protocols}

On arrival at the laboratory, subjects were seated and allowed to rest for 15 minutes, after which blood pressure was recorded. A $10 \mathrm{ml}$ blood sample was then drawn from the contralateral arm after a further five minutes seated rest. Height, body mass, skinfold thicknesses, and waist and hip circumferences were recorded. Dynamic isokinetic leg strength and 10RM were subsequently measured.

\section{Height and body mass}

Height and body mass were recorded using a portable stadiometer and balance weighing scales respectively. Body mass index (BMI) was calculated using the standard formula (mass $(\mathrm{kg})$ divided by height ${ }^{2}(\mathrm{~m})$ ). In addition, waist/hip ratio was calculated using waist circumference, measured at the umbilicus level, and hip girth, measured at the maximum circumference of the buttocks.

\section{Skinfold thickness}

Skinfold thickness was measured, to the nearest $0.5 \mathrm{~mm}$, at four predetermined sites (biceps, triceps, subscapular, and suprailiac) using Harpenden callipers (John Bull, CMS Weighing Equipment Ltd, Camden, London, UK). Skinfold thicknesses were then used to calculate the percentage body fat by the techniques of Durnin and Womersley. ${ }^{24}$ All measurements were taken on the right hand side of the body by the same female experimenter.

\section{Blood pressure}

Subjects were seated and allowed to rest for 15 minutes. Blood pressure was measured using an automatic sphygmomanometer (model 8111; Dynamap 8100T; Critikon, Orange Park, Florida, USA). Mean arterial pressure was calculated using the standard formula: ((systolic pressure-diastolic pressure)/3) + diastolic pressure).

\section{Blood sampling}

A venous blood sample $(10 \mathrm{ml})$ was withdrawn from a prominent superficial vein in the antecubital fossa using a clean venepuncture with minimal stasis. Five millilitres of whole blood was placed in a tube containing a dipotassium ethylenediaminetetra-acetic acid coating and rapidly stored at $-75^{\circ} \mathrm{C}$ for the subsequent determination of blood lipid concentrations. Blood lipid concentrations (TC, HDL-C, and triglyceride) were determined using the Monarch Chemistry System (Instrumentation Laboratories, Warrington, Lancashire, UK) according to the manufacturer's instructions. The assay reagents were supplied by Sigma Diagnostics (St Louis, Missouri, USA). Levels of LDL-C and very low density lipoprotein cholesterol (VLDL-C) were calculated using standard equations..$^{25}$ The remaining $5 \mathrm{ml}$ was placed in a tube containing a lithium heparin coating and spun at $2800 \mathrm{rpm}$ for 10 minutes. Plasma was retained and rapidly stored at $-70^{\circ} \mathrm{C}$ for the subsequent determination of total concentrations of $17 \beta$ oestradiol, luteinising hormone, and follicle stimulating hormone, using a Mini-Vidas System (bioMerieux, Marcy l'Etolie, France) according to the manufacturer's instructions. The assay reagents were supplied by bioMerieux.

\section{Ten repetition maximum}

10RM was assessed for bilateral leg extension and flexion, leg press, bench press, and lat pull down, using weight machines (Power House, Manchester, UK). The weight machines were adjusted to meet the requirements of each subject. Every adjustment was recorded (using the numbered scales on each attachment, for example chair height) and used for all subsequent training and assessment. Subjects performed each exercise to the full extent of their range of motion. 
Table 1 Dynamic isokinetic leg strength over the 16 week test period

\begin{tabular}{|c|c|c|c|c|}
\hline Variable & Treatment & Baseline & After training & After detraining \\
\hline \multirow{2}{*}{$\begin{array}{l}\text { Leg extension (N.m) } \\
(1.04 \mathrm{rad} / \mathrm{s})\end{array}$} & Training & $83.50(21.54)$ & $85.88(21.05)$ & $82.00(22.34)$ \\
\hline & Control & $59.14(18.66)$ & $67.00(20.05)$ & $67.43(20.08)$ \\
\hline \multirow{2}{*}{$\begin{array}{l}\text { Leg flexion (N.m) } \\
(1.04 \mathrm{rad} / \mathrm{s})\end{array}$} & Training & 44.75 (13.26) & $44.00(11.34)$ & $45.25(11.96)$ \\
\hline & Control & $37.71(11.68)$ & 37.43 (12.29) & $39.43(9.38)$ \\
\hline \multirow{2}{*}{$\begin{array}{l}\text { Leg extension (N.m) } \\
(2.09 \mathrm{rad} / \mathrm{s})\end{array}$} & Training & 77.38 (13.44) & $72.50(18.20)$ & $67.88(16.30)$ \\
\hline & Control & $58.43(16.27)$ & $56.57(19.46)$ & $57.43(20.44)$ \\
\hline \multirow{2}{*}{$\begin{array}{l}\text { Leg flexion (N.m) } \\
(2.09 \mathrm{rad} / \mathrm{s})\end{array}$} & Training & $42.88(10.72)$ & $45.00(10.72)$ & $44.13(8.56)$ \\
\hline & Control & 43.00 (10.63) & $33.43(7.18)$ & $35.57(10.98)$ \\
\hline
\end{tabular}

Values are mean (SD)

10RM was taken as the heaviest weight that could be lifted 10 times throughout a complete range of motion. The 10RM was achieved by increasing the load by $5 \mathrm{~kg}$ after each successful set of lifts ( 10 lifts per set) until the maximum load sustainable for 10 lifts was obtained. Subjects were given a five minute rest interval between each weight increment.

\section{Dynamic leg strength}

Before assessment of maximum force, the subjects performed a standardised warm up for five minutes on a cycle ergometer (Monark, Stockholm, Sweden) at $60 \mathrm{rpm}$ with no resistance, and a series of standardised stretches of the quadriceps and hamstring muscles. Dynamic and isometric knee extension and flexion were assessed using a gravity compensated, computer controlled, isokinetic dynamometer (Lido Active; Loredan, Davis, California, USA). Stabilisation straps were secured across the hips and over the shoulders. The ankle cuff was attached around the ankle, proximal to the malleoli. The machine's actuator shaft was aligned with an imaginary transverse line through the femoral condyles. The original knee position was flexion, such that the initial movement was extension. Range of motion (as close to 0 and $90^{\circ}$ (flexion and extension respectively) as possible) was determined and set before the leg was gravity compensated. A resistance pad was placed on the quadriceps, proximal to the patella, to prevent excessive thigh movement.

Subjects performed two submaximal contractions before assessment of maximal voluntary contractions. After three minutes rest, three dynamic maximal voluntary contractions, at angular velocities of 1.04 and $2.09 \mathrm{rad} / \mathrm{s}$, were performed. The greatest peak torque achieved during maximal voluntary contraction was recorded. A one minute rest separated each contraction. Subjects completed this protocol at both velocities using the dominant leg. Standardised verbal encouragement and visual feedback were offered throughout the exercise to promote maximal effort.

\section{Training protocol}

Before training, the 10RM for each exercise was assessed on two occasions separated by 24 hours. Subjects performed a five minute self paced warm up on a cycle ergometer, followed by a series of standardised stretches. They then performed a warm up of 10 repetitions at $50 \%$ of 10RM, for each exercise. l0RM was re-evaluated after four weeks, and training loads adjusted. Subjects performed three sets of eight repetitions at $80 \%$ of their 10RM, with a two minute rest between sets. Subjects cooled down using the same series of stretches that were used for the warm up. In addition to reporting patterns of physical activity, subjects were required to keep training diaries so that the lifting loads could be monitored.

\section{Statistical analysis}

Statistical analyses were performed using a two factor general linear model, incorporating a mixed design, with one between subjects factor of group (two levels: training and control) and one within subjects factor of time (three levels: baseline, after intervention, and detraining). All analyses were corrected for sphericity using the methods of Greenhouse and Geisser. ${ }^{25 a}$ Statistical significance was accepted at the $\mathrm{p}<0.05$ level.

Post hoc analyses were conducted using paired $t$ tests to examine differences between resting values and those after eight weeks of training and a further eight weeks of detraining. Bonferroni correction was applied meaning that statistical significance was accepted at the $\mathrm{p}<0.0166$ level.

\section{RESULTS}

At baseline, there were no significant differences between groups for any strength measure. Statistical analysis showed significant group by time interactions for lat pull down $\left(F_{1,13}=\right.$ $16.60 ; \mathrm{p}<0.005)$, bench press $\left(F_{1,13}=13.79 ; \mathrm{p}<0.01\right)$, leg press $\left(F_{1,13}=15.65 ; \mathrm{p}<0.01\right)$, and knee extension $\left(F_{1,13}=12.60\right.$; $\mathrm{p}<0.01)$ 10RM strength. However, no significant interaction was observed for 10RM knee flexion $\left(F_{1,13}=0.87 ; \mathrm{p}=0.37\right)$. Further examination of the significant interactions using paired $t$ tests showed that eight weeks of resistance training resulted in significant increases in 10RM strength in the training group compared with controls (fig l). Lat pull down increased by $88 \%(p<0.005)$, bench press by $120 \%(p<0.005)$, leg press by $80 \%(p<0.005)$, and knee extension by $650 \%$ $(\mathrm{p}<0.005)$ in the training group. Although 10RM strength decreased after eight weeks of detraining, results remained significantly elevated from baseline measures in the training group for lat pull down $(p<0.005)$, bench press $(p<0.005)$, leg press $(p<0.005)$, and knee extension $(p<0.01)$. Although eight weeks of training resulted in significant changes in 10RM strength, there were no significant differences in isokinetic knee extension or flexion tests, when measured at either 1.04 or $2.09 \mathrm{rad} / \mathrm{s}$ (table 1 ).

There were no significant time or group main effects for TC, HDL-C, LDL-C, VLDL-C, TC/LDL-C ratio, TC/HDL-C ratio or triglyceride concentrations, nor were there any significant time by group interactions (table 2 ). This illustrates that blood lipid concentrations were unaffected by eight weeks of resistance training at $80 \%$ of $10 \mathrm{RM}$. The data also show that after training there were no significant alterations in body composition, as indicated by body mass, waist to hip ratio, percentage body fat, or BMI (table 3).

The results indicate that resistance training had no significant effect on blood pressure (table 4). The effect size for changes in systolic, diastolic, and mean arterial blood pressure in the training group was $0.473,0.63$, and 0.513 respectively. A closer examination of the data indicated that the training group exhibited a trend towards a reduction in systolic $(11.03 \%, p=0.139)$, diastolic $(8.5 \%, p=0.059)$, and mean arterial $(9.7 \%, \mathrm{p}=0.157)$ blood pressures, with concomitant increases back towards baseline values after eight weeks of detraining. No such trend was observed in the control group. 
Table 2 Blood lipid profiles over the 16 week test period

\begin{tabular}{|c|c|c|c|c|}
\hline Variable & Treatment & Baseline & After training & After detraining \\
\hline \multirow[t]{2}{*}{ TC (mmol/l) } & Training & $6.94(1.66)$ & $6.50(1.22)$ & $5.94(1.09)$ \\
\hline & Control & $6.62(1.21)$ & 6.77 (1.37) & $6.54(1.05)$ \\
\hline \multirow[t]{2}{*}{ HDL-C (mmol/I) } & Training & $1.99(0.37)$ & $2.01(0.32)$ & $2.04(0.40)$ \\
\hline & Control & $1.85(0.38)$ & $1.96(0.37)$ & $1.59(0.48)$ \\
\hline \multirow[t]{2}{*}{ LDL-C (mmol/I) } & Training & 4.23 (1.69) & $4.12(1.35)$ & $3.29(0.87)$ \\
\hline & Control & $4.45(0.82)$ & $4.43(1.52)$ & $4.08(1.21)$ \\
\hline \multirow[t]{2}{*}{ TC/HDL-C ratio } & Training & $3.71(1.56)$ & $3.38(1.17)$ & $3.08(1.11)$ \\
\hline & Control & $3.89(1.00)$ & $3.32(0.86)$ & 4.48 (1.69) \\
\hline \multirow[t]{2}{*}{ TC/LDL-C ratio } & Training & $1.56(0.28)$ & $1.63(0.24)$ & $1.71(0.27)$ \\
\hline & Control & $1.46(0.12)$ & $1.58(0.32)$ & $1.45(0.17)$ \\
\hline \multirow[t]{2}{*}{ VLDL-C (mmol/l) } & Training & $0.36(0.30)$ & $0.34(0.15)$ & $0.27(0.09)$ \\
\hline & Control & $0.31(0.16)$ & $0.38(0.23)$ & $0.38(0.25)$ \\
\hline \multirow[t]{2}{*}{ Triglycerides (mmol/l) } & Training & $1.81(1.54)$ & $1.68(0.76)$ & $1.37(0.47)$ \\
\hline & Control & $1.53(0.80)$ & $1.92(1.14)$ & $1.87(1.27)$ \\
\hline
\end{tabular}

Table 3 Body composition over the 16 week test period

\begin{tabular}{lllll}
\hline Variable & Treatment & Baseline & After training & After detraining \\
\hline Body mass $(\mathrm{kg})$ & $\begin{array}{l}\text { Training } \\
\text { Control }\end{array}$ & $\begin{array}{l}68.41(13.06) \\
59.99(7.69)\end{array}$ & $\begin{array}{l}67.75(12.99) \\
60.36(7.44)\end{array}$ & $\begin{array}{l}68.00(12.68) \\
59.86(7.27)\end{array}$ \\
& & & & \\
Waist/hip ratio & Training & $0.81(0.08)$ & $0.80(0.08)$ & $0.81(0.09)$ \\
& Control & $0.80(0.04)$ & $0.79(0.04)$ & $0.80(0.03)$ \\
Body mass index & Training & $26.89(5.94)$ & $26.63(5.92)$ & $26.74(5.87)$ \\
& Control & $23.97(2.58)$ & $24.13(2.53)$ & $23.92(2.39)$ \\
\% Body fat & Training & $30.0(3.0)$ & $30.5(3.0)$ & $31.0(2.0)$ \\
& Control & $28.0(4.0)$ & $28.5(4.0)$ & $28.5(5.0)$ \\
\hline
\end{tabular}

Values are mean (SD).

Table 4 Blood pressure over the 16 week test period

\begin{tabular}{lllll}
\hline Variable & Treatment & Baseline & After training & After detraining \\
\hline Systolic $(\mathrm{mm} \mathrm{Hg})$ & Training & $133(20)$ & $118(15)$ & $129(14)$ \\
& Control & $133(20)$ & $132(15)$ & $134(15)$ \\
Diastolic $(\mathrm{mm} \mathrm{Hg})$ & Training & $72(11)$ & $66(10)$ & $67(11)$ \\
& Control & $75(10)$ & $78(11)$ & $77(9)$ \\
Mean arterial pressure & Training & $92(13)$ & $83(10)$ & $88(11)$ \\
(mm Hg) & Control & $94(11)$ & $96(10)$ & $96(10)$ \\
\hline
\end{tabular}

Values are mean (SD).

\section{DISCUSSION}

The results show significant increases in 10RM strength for exercises involving arm and leg muscles in postmenopausal women. The improvements in strength may have resulted from muscle hypertrophy, although this cannot be confirmed, as muscle cross sectional area was not measured. These findings show that a short duration, low intensity resistance training programme produced substantial improvements in muscle strength. Perhaps the most important benefit of increased muscle strength for postmenopausal women is a reduced risk of falling and sustaining injury. ${ }^{26}$ Improved strength may also aid functional activities such as rising from a low chair and climbing stairs.' Resistance training programmes also have secondary general health benefits, including reversal of functional decline due to age, ${ }^{27}$ prevention and management of osteoporosis, ${ }^{28}$ and relief from arthritis. ${ }^{29}$ Supervised exercise may also have psychosocial advantages such as alleviation of feelings of depression, loneliness, and isolation and improvements in cognitive function. ${ }^{29}$

The resistance training programme used in this study was ideally suited to postmenopausal women. Based on previous research, subjects trained three times a week, on nonconsecutive days, ${ }^{30}$ performing three sets of each exercise at 
$80 \%$ of $10 \mathrm{RM}$. Although this intensity is equivalent to $60 \%$ of $1 \mathrm{RM}^{32}$ the use of $10 \mathrm{RM}$ to set work intensity was considered more appropriate in this sample as subjects were elderly and previously sedentary. This concession is especially pertinent during the initial stages of a progressive weight training programme, when considerable learning is taking place.

Despite significant improvements in lORM strength with training, there were no significant changes in maximal dynamic leg strength. Lexell et $a l^{30}$ observed that isokinetic strength did not increase to the same magnitude as dynamic IRM strength after a heavy resistance training programme in elderly women. Several authors have reported similar results. ${ }^{31-34}$ Abernethy et al ${ }^{34}$ suggested that when differences in strength after resistance training are measured, the mode of assessment is influential as strength tests differ in terms of movement patterns, contractile speeds, and loading modalities. The modality of the strength test will determine which neuromuscular qualities are assessed. Therefore, any potential differences in maximal dynamic isokinetic strength in this study may have been masked by an insensitive strength test.

The current training programme failed to evoke any significant alterations in blood lipid profiles. Similarly, Kokkinos et $a l^{35}$ reported that strength training did not improve lipoprotein lipid profiles in middle aged men. However, the findings of this study are in contrast with those of Bemben and Bemben, ${ }^{22}$ who found improvements in HDL-C concentrations and TC/HDL-C ratio after a 16 week Dynaband resistance exercise programme in postmenopausal women. Prabhakaran et al $l^{18}$ also observed significant reductions in TC, LDL-C and TC/HDL-C ratio in a group of resistance trained women. However, these studies examined the effects of more prolonged ( 16 and 14 weeks respectively) and intense (Prabhakaran et al ${ }^{18}$ trained subjects at $85 \%$ of lRM) training programmes. Prabhakaran et $a l^{18}$ were able to use a higher work intensity as they recruited a younger (mean age about 27 years) group of premenopausal women. However, they did not elicit changes in either HDL-C or triglyceride concentrations. Previous findings have suggested that exercise that fails to generate weight $\operatorname{loss}^{36}$ or the expenditure of more than $1100 \mathrm{kcal}(4.6 \mathrm{MJ})$ of energy $^{37}$ per exercise session will not affect HDL-C levels. No significant differences in body mass, BMI, waist/hip ratio, or percentage body fat were found after eight weeks of resistance training in this study. The results indicate that, whereas changes in muscle strength occur quite rapidly, changes in blood lipid profile occur at a lower rate and are independent of changes in muscle strength.

One of the reported benefits of increased physical activity for postmenopausal women is a reduction in blood pressure (high blood pressure is an independent cardiovascular risk factor).$^{38}$ Although not significant, our data showed a trend towards a reduction in systolic blood pressure, diastolic blood pressure, and mean arterial pressure in the resistance trained group compared with controls. It should be noted that blood pressure after training decreased from values classified as high normal to values classified as optimal according to World Health Organisation guidelines. ${ }^{39}$ There is evidence that reductions in blood pressure of the magnitude shown in this study are associated with a significant decline in the risk of both stroke and ischaemic heart disease. ${ }^{38}$ This indicates that, although the decreases in blood pressure were not statistically significant, they may have clinical or biological significance. However, because of the dropout rate of our subjects, there was a relatively low statistical power attributed to the group by time interaction term (0.31). This is suggested as a possible reason for the non-significant findings for blood pressure. Thus, the possibility of committing a type II error must be considered when drawing conclusions from the blood pressure results of this study.

In conclusion, the training programme used in this study produced significant increases in 10RM strength, but failed to produce significant changes in blood lipid profiles, body com-

\section{Take home message}

Eight weeks of low intensity resistance training produces substantial improvements in muscle strength in sedentary postmenopausal women. We recommend that this programme should be used as the initial stage of a longer, progressive resistance training programme.

position, or blood pressure. Therefore we conclude that a low intensity, short duration resistance training programme should form the initial stage of a progressive resistance training programme for sedentary postmenopausal women. It may be that a longer training programme at higher work intensity is needed to evoke significant changes in blood lipid concentrations, blood pressure, or body composition. To examine the cumulative effects of HRT and resistance exercise, future research should also compare training in HRT users and non-HRT users.

\section{Authors' affiliations}

K J Elliott, C Sale, N T Cable, Research Institute for Sport and Exercise Sciences, Liverpool John Moores University, Liverpool, UK

\section{REFERENCES}

1 Phillips SK, Rook KM, Siddle NC, et al. Muscle weakness in women occurs at an earlier age than in men, but is preserved by hormone replacement therapy. Clin Sci 1993;84:95-8.

2 Rutherford OM, Jones DA. The relationship of muscle and bone loss and activity levels with age in women. Age Ageing 1992:21:286-93.

3 Nelson ME, Fiatarone MA, Morganti CM, et al. Positive effects of high intensity strength training on multiple risk factors for osteoporotic fractures. JAMA 1994;272:1909-14

4 Larsson L. Histochemical characteristics of human skeletal muscle during aging. Acta Physiol Scand 1983;117:469-71.

5 Greeves JP, Cable NT, Reilly T, et al. Changes in muscle strength in women following the menopause: a longitudinal assessment of the efficacy of hormone replacement therapy. Clin Sci 1999;97:79-84.

6 Skelton DA, Phillips SK, Bruce SA, et al. Hormone replacement therapy increases isometric muscle strength of adductor pollicis in post-menopausal women. Clin Sci 1999;96:357-64.

7 Dornemann TM, McMurray RG, Renner JB, et al. Effects of high-intensity resistance exercise on bone mineral density and muscle strength of 40-50-year-old women. J Sports Med Phys Fitness 1997;37:246-51.

8 Rhodes EC, Martin AD, Taunton JE, et al. Effects of one year of resistance training on the relation between muscular strength and bone density in elderly women. Br J Sports Med 2000;34: 18-22

9 Taaffe DR, Pruitt L, Pyka G, et al. Comparitive effects of high- and low-intensity resistance training on thigh muscle strength, fiber area, and tissue composition in elderly women. Clin Physiol 1996;16:381-92.

10 British National Formulary. London: British Medical Association and the Royal Pharmaceutical Society of Great Britain, 2001

11 Lip GY, Blann AD, Jones AF, et al. Effects of hormone replacement therapy on homeostatic factors, lipid factors and endothelial function in women undergoing surgical menopause: implications for prevention of arteriosclerosis. Am Heart J 1997;134:764-71.

12 Sacks FM, Walsh BW. The effects of reproductive hormones on serum lipoproteins: unresolved issues in biological and clinical practice. Ann N Y Acad Sci 1990;592:272-85

13 Krauss RM. Lipids and lipoproteins and effects of hormone replacement. In: Lobo RA, ed. Treatment of the post-menopausal women: basic and clinical aspects. New York: Raven Press, 1994.

14 Sacks FM, Walsh BW. Sex hormones and lipoprotein metabolism. Curr Opin Lipidol 1994;5:236-40.

15 Blair SN, Kampert JB, Kohl HW, et al. Influences of cardiorespiratory fitness and other precursors on cardiovascular disease and all-cause mortality in men and women. JAMA 1996;17:205-10.

16 Kushi LH, Fee RM, Folsom AR, et al. Physical activity and mortality in postmenopausal women. JAMA 1997:277:1287-92.

17 Lindheim SR, Notelovitz M, Feldman EB, et al. The independent effects of exercise and estrogen on lipids and lipoproteins in postmenopausal women. Obstet Gynecol 1994;83:167-72.

18 Prabhakaran B, Dowling EA, Branch JD, et al. Effect of 14 weeks of resistance training on lipid profile and body fat percentage in premenopausal women. Br J Sports Med 1999:33:190-5.

19 Grundy SM. Cholesterol and coronary heart disease. Arch Intern Med 1997; 157: 1 177-84.

20 Skinner ER. Lipid metabolism. Curr Opin Lipidol 1996;7:U1 19-24.

21 Vega GL, Sirtori C. Dyslipidemia and coronary heart disease. Curr Opin Lipidol 1996;7:179-82. 
22 Bemben DA, Bemben MG. Effects of resistance exercise and body mass index on lipoprotein-lipid patterns of postmenopausal women. J Strength Cond Res 2000;14:80-6.

23 Lamb KL, Brodie DA. Leisure-time physical activity as an estimate of physical fitness: a validation study. J Clin Endocrinol 1991:44:41-52.

24 Durnin JVGA, Womersley, J. Body fat assessed from total body density and its estimation from skinfold thickness: measurements on 481 men and women aged from 16-72 years. Br J Nutr 1974;32:77.

25a Vincent WJ. Statistics in kinesiology. 2nd ed. Leeds: Human Kinetics, 1999.

25 Friedwald WT, Levy RI, Fredrickson DS. Estimation of the concentration of low-density lipoprotein cholesterol in plasma without use of the preparation of ultracentrifuge. Clin Chem 1972;18:499-502.

26 Province MA, Hadley EC, Hornbrook MC, et al. The effects of exercise on falls in elderly patients: a pre-planned meta-analysis of the FICSIT trials. JAMA 1995;273:1341-7.

27 Skelton DA, Mclaughlin AW. Training functional ability in old age. Physiotherapy 1996;82:159-67.

28 Snow-Harter C, Bouxsein ML, Lewis BT, et al. Effects of resistance and endurance exercise on bone mineral status of young women: a randomised exercise intervention trial. J Bone Miner Res 1992;7:761-9.

29 Rai D, Finch H. Physical activity 'from our point of view': qualitative research among South Asian and black communities. London: Health Education Authority, 1997.

30 Lexell J, Downham DY, Larsson Y, et al. Heavy-resistance training in older Scandinavian men and women: short- and long-term effects on arm and leg muscles. Scand J Med Sci Sports 1995;5:329-41.
31 Brown AB, McCartney N, Sale DG. Positive adaptations to weight lifting in the elderly. J Appl Physiol 1990;69:1725-33.

32 Frontera WR, Meredith CN, O'Reilly KP, et al. Strength conditioning in older men: skeletal hypertrophy and improved function. J Appl Physiol 1988;64: 1038-44

33 Grimby G, Aniansson A, Hedberg $M$, et al. Training can improve muscle strength and endurance in 78- to 84-year-old men. J Appl Physiol 1992;73:2517-23.

34 Abernethy PJ, Wilson G, Logan P. Strength and power assessment. Sports Med 1995;19:401-17

35 Kokkinos PF, Hurley BF, Smutok MA, et al. Strength training does not improve lipoprotein-lipid profiles in men at risk for CHD. Med Sci Sports Exerc 1991;23:1134-9.

36 Fonong $\mathrm{T}$, Toth $\mathrm{M}$, Ades PA, et al. Relationship between physical activity and HDL-cholesterol in healthy older men and women: a cross-sectional and exercise intervention study. Atherolsclerosis 1996; 127:177-83.

37 Ferguson MA, Alderson NL, Trost SG, et al. Effects of four different single exercise sessions on lipids, lipoproteins, and lipoprotein lipase. J Appl Physiol 1998;85:1169-74.

38 Stamler J, Stamler R, Neaton JD. Blood pressure, systolic and diastolic, and cardiovascular risks. Arch Intern Med 1993;153:598-615.

39 Guidelines Subcommittee. 1999 World Health

Organisation-International Society of Hypertension guidelines for the management of hypertension. J Hypertens 1999;17:151-83.

\section{British Association of Sport and Exercise Medicine in association with the National Sports Medicine Institute}
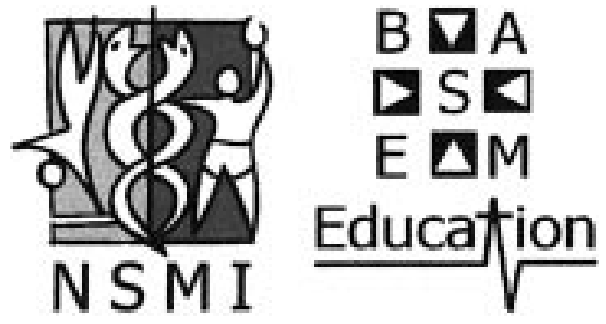

Education programme 2002

General Sports Medicine

Lilleshall National Sports Centre, 22-27 September

Practical Sport and Medicine Meeting

Club La Santa, Lanzarote (families \& non-delegates welcome), 3-10 October

Diploma Preparation

Location and date to be confirmed, October

The Queen's Golden Jubilee \& Post Commonwealth Games BASEM Congress

The Low Wood Hotel and Conference Centre, Windermere,

10-13 October
Intermediate Sports Injury Management and Medicine-Lower Limb Lilleshall National Sports Centre, 17-22 November

Current Concepts

Topic, location, and date to be confirmed, December

\section{Education programme 2003}

Intermediate Sports Injury Management and Medicine-Head, Neck, and Upper Limb

Lilleshall National Sports Centre, 23-28 February

General Sports Medicine

Lilleshall National Sports Centre, 27 April-2 May Diploma Preparation Course

Sheffield Centre of Sports Medicine, May

\section{Current Concepts}

Topic, location, and date to be confirmed, May

Intermediate Sports Injury Management and Medicine-Lumbar Spine, Thorax, Groin, Pelvis, and Hip

Lilleshall National Sports Centre, 6-11 July

General Sports Medicine

Lilleshall National Sports Centre, 21-26 September

For further details of these courses please contact Mr Barry Hill, The National Sports Medicine Institute, 32 Devonshire Street, London WIG 6PX, UK. Tel: 0207908 3642; Fax: 0207908 3635; email: barry.hill@nsmi.org.uk; www.nsmi.org.uk 